\title{
Editorial
}

\section{Hypoxia-Inducible Factor 1 a (HIF-1a), Angiopoietin-2 (ANG-2) and Endocan: Novel Biomarkers of Disease Progression Involving Polycystic Kidney Disease}

\author{
Frederic F. Rahbari-Oskoui ${ }^{\mathrm{a}}$ Arlene B. Chapman ${ }^{\mathrm{b}}$ \\ a Department of Medicine, Emory University School of Medicine, Atlanta, GA, USA; ${ }^{b}$ Department of Medicine, \\ University of Chicago Medicine, Chicago, IL, USA
}

Discovery and validation of biomarkers comprise a critical step in making progress in the fight against autosomal dominant polycystic kidney disease (ADPKD). Biomarkers can be on 3 different levels: diagnostic, prognostic, and therapeutic. The implementation of these biomarkers in the design and feasibility of clinical trials depends on their accuracy, reproducibility and dynamic response to an intervention.

Two novel imaging markers, renal artery blood flow (RABF) and height-adjusted total kidney volume (HtTKV), are considered prognostic biomarkers in ADPKD [1-3]. HtTKV was approved by the United States Food and Drug Administration in 2014 for use as an enrichment biomarker in clinical trials. Change in HtTKV, used as the primary endpoint in clinical trials, has been associated with an unprecedented enthusiasm for interventional trials in ADPKD over the past 15 years, leading to the marketing of the first disease-modifying agent (tolvaptan) outside of the United States [4]. A predictive model utilizing HtTKV, age, sex, race, and serum creatinine has been developed as a risk stratification tool for disease progression in ADPKD [5]. Clinical trial de- sign utilizing the Irazabal classification system allows for the enrollment of "fast progressing" patients, making clinical trial design shorter with fewer subjects ultimately being less expensive and less burdensome on the patient population.

Both HtTKV and RABF require an MRI or MRA, which remain cumbersome, expensive (if it cannot be performed in resource-limited countries), and technically difficult to obtain (especially for RABF). Therefore, a continued search for more accurate biomarkers of disease progression has gained a significant momentum over the past decade. These biomarkers include urinary concentrations of copeptin, mitochondrial pyruvate carrier (MPC-1), neutrophil gelatinase-associated lipocalin, interleukin 8 , heart-type-fatty acid binding protein, kidney injury molecule 1 , and serum levels of high density lipid cholesterol, vascular endothelial growth factor (VEGF), angiopoietin-1, uric acid levels and MPC-1, and tumor necrosis factor alpha [6].

In this issue of the journal, Raptis and colleagues report the first human comparison of the serum levels of 3 novel biomarkers of endothelial function (endocan also (c) 2018 S. Karger AG, Basel

E-Mail karger@karger.com

www.karger.com/ajn
Arlene Chapman, MD

Department of Medicine, University of Chicago 5841 S. Maryland Ave.

MC 5100, Chicago, IL 60637 (USA)

E-Mail Achapman1@ medicine.bsd.uchicago.edu 
called Endothelial cell-specific molecule 1 or ESM-1), angiogenesis (angiopoietin-2), and renal hypoxia (HIF-1 $\alpha$ ) [7]. They evaluated 26 normal controls, 26 patients with early (estimated glomerular filtration rate [eGFR] $>70$ $\mathrm{mL} / \mathrm{min}$ ) and 26 with more advanced ADPKD (GFR 45$70 \mathrm{~mL} / \mathrm{min}$ ) [7]. The authors show significantly higher serum values of all 3 biomarkers in ADPKD patients with more advanced compared to both less advanced and normal controls, suggesting that these molecules may represent early markers of disease progression in ADPKD. Furthermore, they show modest to moderate correlations between eGFR and endocan $(r=-0.258)$, angiopoietin-2, and HIF- $1 \alpha$ ( $r=-0.468$ and -0.465 respectively) levels. Even more intriguing is the fact that they demonstrate strong correlations between the ADMA levels (an established marker of endothelial dysfunction in ADPKD) and endocan ( $r=0.908)$, angiopoietin-2 $(r=0.983)$, and HIF$1 \alpha(r=0.998)$ levels. The authors speculate on the role of potential intermediary molecules that may link these distinct pathways together (VEGF and nitrogen oxide).

While this report is limited by its sample size, the authors pose legitimate questions, generate new hypotheses, and offer practical implications on the direction of future clinical and translational research in ADPKD.

The addition of HIF-1a signaling pathway to the previously reported correlation between $\mathrm{RABF}$ and renal function raises an interesting question: Is the decline in GFR that follows the fall in RABF mediated through pure hemodynamic changes without hypoxia, through hypoxia, or both? Investigating the relationship between these 2 factors, may lead to a better understanding of the disease progression in the early stages of ADPKD.

Similarities that exist between ADPKD tubular epithelial cell behavior and neoplastic cell behavior (de-differentiation, abnormal trafficking, and targeting of proteins such as the epidermal growth factor receptor, polarization defects, increased cell-matrix and reduced cell-cell adhesion, high rates of division, and apoptosis), explain the recent rise of interest in investigating clinical pathways or molecules that have shown some relevance in oncology (bosutinib, tesevatinib, and others) [8]. This report adds specific molecules (HIF-1a, endocan, and angiopoietin 2) that are involved in 2 critical steps of neoplastic process: hypoxia and angiogenesis. Furthermore, it links these molecules to a previously known marker of endothelial dysfunction, asymmetric dimethylarginine (ADMA). The strong correlation between ADMA and the 3 novel biomarkers, belonging to 3 distinct signaling pathways, may be suggestive of either a central place (through unknown intermediary molecules, may be nitric oxide, or VEGF) or a very distal (downstream) place for ADMA in those pathways. Only future studies may elucidate that point.

However, our experience with previously identified candidate biomarkers of disease progression has not yet made any significant impact on clinical practice or research, and thus points out the difficulties of developing useful biomarkers in ADPKD. As a reminder, HtTKV was first shown to predict disease progression in ADPKD approximately 12 years ago, and approval of this tool for clinical trial enrichment took more than a decade to come to fruition. The lessons learned during the TKV validation (choosing the most reproducible imaging tool to measure TKV, combining collaborative efforts from large multinational registries, using longitudinal predictive power rather than cross-sectional correlations, etc.) will need to be applied to these biomarkers to accelerate their validation.

Several issues need to be resolved to help in the validation process. Establishing a robust reference range for normal values (in the general population, chronic kidney disease patients, and ADPKD patients), using a standardized assay and reproducible assay with known coefficients of variation will be important. Published data include different methodologies, different biological fluids (serum vs. plasma), and different assay-to-assay variability in different cohorts. Given the paucity of results in chronic kidney disease (CKD) or ADPKD cohorts, direct comparisons between those levels are not available to determine the direct applicability to ADPKD. Reported mean values of plasma Endocan in this study $(4.85 \mu \mathrm{g} / \mathrm{dL}$ using the Cusabio assay) are 7 -fold higher than the previously published serum levels in a Turkish cohort (control group for a cohort of patients with Behçet disease; mean $0.7 \mu \mathrm{g} / \mathrm{dL}$ using the Bio-Tek Synergy HT assay; Balta et al. [9]) but similar to the plasma levels in an Egyptian cohort $(4.12 \pm 2.9 \mu \mathrm{g} / \mathrm{dL}$ using enzyme-linked immunosorbent assay, LUNGINNOV Systems assay) [10]. Whether the observed differences are simply due to cohort differences, biological sample differences, laboratory methodology, or assay-to-assay variations is unclear. Furthermore, the factors that lead to day-to-day variations and loss of reproducibility need to be identified and adjusted for.

Clinically what level of incremental changes in concentration associates with and/or is predictive of relevant outcomes (doubling of serum creatinine levels, progression to CKD-stages III-IV, time to onset of end-stage renal disease, or death) remains to be determined. This paper shows a significant but moderate correlation between 
eGFR and biomarkers and more needs to be done. Also, any future prognostic model that includes several parameters with strong correlation to each other (ADMA vs. HIF-1 $\alpha$, Angiopoietin 1 and endocan) should be carefully investigated for collinearity and for a more excitingly true biological relevance.

The field of biomarker development will need to address the equipoise of current knowledge and will be useful only if they enhance the robustness of existing biomarkers, or if they are less expensive. To illustrate this point, the Consortium for Radiologic Imaging Studies in PKD looking at independent prognostic value of HtTKV vs. PKD genotype or mutation type showed that both PKD genotype and mutation type and HtTKV-predicted CKD outcomes, $\mathrm{PKD}$ genotype, or mutation type was not an independent prognostic factor after adjusting for HtTKV [11].

The exciting additional discovery of new biomarkers in ADPKD that sheds light on potential new mechanistic pathways involved in cyst proliferation, fluid secretion, and renal injury opens new doors to test new interventions. Raptis et al. [7] targeted endothelial dysfunction, angiogenesis, and renal hypoxia. These efforts will potentially bring new molecules into clinical trials and lead to new promising therapeutic interventions for ADPKD.

\section{Disclosure Statement}

Both authors declared that they have no conflicts of interest to disclose.

\section{References}

1 King BF, Torres VE, Brummer ME, Chapman AB, Bae KT, Glockner JF, Arya K, Felmlee JP, Grantham JJ, Guay-Woodford LM, Bennett WM, Klahr S, Hirschman GH, Kimmel PL, Thompson PA, Miller JP; Consortium for Radiologic Imaging Studies of Polycystic Kidney Disease (CRISP): Magnetic resonance measurements of renal blood flow as a marker of disease severity in autosomal-dominant polycystic kidney disease. Kidney Int 2003;64: 2214-2221.

-2 Grantham JJ, Torres VE, Chapman AB, Guay-Woodford LM, Bae KT, King BF Jr, Wetzel LH, Baumgarten DA, Kenney PJ, Harris PC, Klahr S, Bennett WM, Hirschman GN, Meyers CM, Zhang X, Zhu F, Miller JP; CRISP Investigators: Volume progression in polycystic kidney disease. N Engl J Med 2006;354: 2122-2130.

-3 Torres VE, King BF, Chapman AB, Brummer ME, Bae KT, Glockner JF, Arya K, Risk D, Felmlee JP, Grantham JJ, Guay-Woodford LM, Bennett WM, Klahr S, Meyers CM, Zhang X, Thompson PA, Miller JP; Consortium for Radiologic Imaging Studies of Polycystic Kidney Disease (CRISP): Magnetic resonance measurements of renal blood flow and disease progression in autosomal dominant polycystic kidney disease. Clin J Am Soc Nephrol 2007;2:112-120.

- 4 Torres VE, Chapman AB, Devuyst O, Gansevoort RT, Grantham JJ, Higashihara E, Perrone $\mathrm{RD}$, Krasa HB, Ouyang J, Czerwiec FS; TEMPO3:4TrialInvestigators:Tolvaptaninpatients with autosomal dominant polycystic kidneydisease. NEnglJMed 2012;367:2407-2418.

5 Irazabal MV, Rangel LJ, Bergstralh EJ, Osborn SL, Harmon AJ, Sundsbak JL, Bae KT, Chapman AB, Grantham JJ, Mrug M, Hogan MC, El-Zoghby ZM, Harris PC, Erickson BJ, King BF, Torres VE; CRISP Investigators: Imaging classification of autosomal dominant polycystic kidney disease: a simple model for selecting patients for clinical trials. J Am Soc Nephrol 2015;26:160-172.

- 6 Schrier RW, Brosnahan G, Cadnapaphornchai MA, Chonchol M, Friend K, Gitomer B, Rossetti S: Predictors of autosomal dominant polycystic kidney disease progression. J Am Soc Nephrol 2014;25:2399-2418.

-7 Raptis V, Bakogiannis C, Loutradis C, Boutou A, Lampropoulou I, Intzevidou E, Sioulis A, Balaskas E, Sarafidis P: Levels of endocan, angiopoietin-2 and hypoxia-inducible factor- $1 \mathrm{a}$ in patients with autosomal dominant polycystic kidney disease and different levels of renal function. Am J Nephrol 2018;47:231-238.

$\checkmark 8$ Torres VE, Harris PC: Mechanisms of disease: autosomal dominant and recessive polycystic kidney diseases. Nat Clin Pract Nephrol 2006; 2:40-55; quiz 55.

-9 Balta I, Balta S, Koryurek OM, Demirkol S, Mikhailidis DP, Celik T, Cakar M, Kucuk U, Eksioglu M, Kurt YG: Serum endocan levels as a marker of disease activity in patients with Behçet disease. J Am Acad Dermatol 2014;70: 291-296.

10 Abd El Halim A, Sayed M: Serum endocan role in diagnosis and prognosis of ventilator associated pneumonia. Egypt J Chest Dis Tubercu 2015;64:865-869.

-11 Yu ASL, Shen C, Landsittel DP, Harris PC, Torres VE, Mrug M, Bae KT, Grantham JJ, Rahbari-Oskoui FF, Flessner MF, Bennett WM, Chapman AB; Consortium for Radiologic Imaging Studies of Polycystic Kidney Disease (CRISP): Baseline total kidney volume and the rate of kidney growth are associated with chronic kidney disease progression in Autosomal Dominant Polycystic Kidney Disease. Kidney Int 2018;93:691-699. 